\title{
Insulin metabolism markers are predictors of subclinical atherosclerosis among overweight and obese children and adolescents
}

\author{
Golaleh Asghari ${ }^{1}$, Pooneh Dehghan ${ }^{2}$, Parvin Mirmiran ${ }^{3 *}$, Emad Yuzbashian $^{4}$, Maryam Mahdavi ${ }^{5}$, Maryam Tohidi ${ }^{6}$, \\ Tirang R. Neyestani ${ }^{7}$, Farhad Hosseinpanah ${ }^{8^{*}}$ and Fereidoun Azizi ${ }^{9}$
}

\begin{abstract}
Background: To investigate the association between markers of insulin metabolism and carotid intima-media thickness(CIMT) among overweight and obese children and adolescents.

Methods: A total of 378 children and adolescents aged from 6 to 13 years, with WHO body mass index Z-Scores $\geq 2$ were enrolled in this study. We measured fasting serum insulin and glucose, conducted a homeostatic model assessment of insulin resistance(HOMA-IR), and calculated the quantitative insulin sensitivity check index(QUICKI). Carotid intima-media thickness was measured in the common carotid artery with high-resolution ultrasonography.

Results: The study participants consisted of 198 boys and 180 girls with a mean( \pm SD) age of $9.3 \pm 1.7$ years, 18.3\% being pre-pubertal. In boys, after controlling for confounders, a one-SD increase in fasting insulin and HOMA-IR were associated with $0.351 \mathrm{~mm}(P<0.001)$ and $0.350 \mathrm{~mm}(P<0.001)$ increases in $\mathrm{CIMT}$, respectively. However, a oneSD increase in QUICKI was associated with a $-0.305 \mathrm{~mm}(P=0.001)$ decrease in CIMT. When categorizing into tertiles, a one-SD increase in fasting insulin and HOMA-IR were associated with 87 and $81 \%$ increases in the odds of higher categories of CIMT(both $P<0.05$ ). However, a one-SD increase in QUICKI was associated with 37\% lower odds of higher categories of $\operatorname{CIMT}(P=0.022)$. No significant associations were found among girls.

Conclusion: This study demonstrated that insulin resistance and sensitivity markers were independent predictors of CIMT in overweight and obese boys, but not in girls, highlighting the importance of chronically elevated insulin levels for predisposing these boys to alterations in their vascular structure.
\end{abstract}

Keywords: Insulin resistance, Atherosclerosis, Children, Adolescents, obesity

\section{Background}

Obesity-related insulin resistance and chronically elevated insulin concentrations that begin in childhood can induce atherothrombotic mechanisms, reduce fibrinolytic balance, and impair endothelial function, which independently contributes to future cardiovascular events

\footnotetext{
*Correspondence: mirmiran@endocrine.ac.ir; parvin.mirmiran@gmail.com; fhospanah@endocrine.ac.ir

${ }^{3}$ Nutrition and Endocrine Research Center, Research Institute for Endocrine Sciences, Shahid Beheshti University of Medical Sciences, P.O. Box:

19395-4763, Tehran, Iran

${ }^{8}$ Obesity Research Center, Research Institute for Endocrine Sciences, Shahid Beheshti University of Medical Sciences, P.O. Box: 19395-4763, Tehran, Iran Full list of author information is available at the end of the article
}

in adulthood $[1,2]$. Atherosclerosis develops gradually, over the course of a lifetime, and a long crucial phase of the process consists of several silent dysfunctional changes of the endothelium [3]. The increased mortality associated with atherosclerosis is related to the duration and intensity of risk factors presenting throughout a lifetime. Therefore, to prevent cardiovascular disease from manifesting and known factors leading to clinical events, it is important to unravel which factors play a role in the earlier and later stages of the atherosclerotic disease.

In recent years, high-resolution $\mathrm{B}$ mode ultrasonography has been frequently used in children and adolescents to measure carotid intima-media thickness (cIMT)

(c) The Author(s). 2018 Open Access This article is distributed under the terms of the Creative Commons Attribution 4.0 International License (http://creativecommons.org/licenses/by/4.0/), which permits unrestricted use, distribution, and 
for the purpose of assessing the presence of subclinical atherosclerosis. Atherosclerotic monitoring with IMT is both sensitive and reproducible, providing a surrogate measure of atherosclerotic burden and cardiovascular risk which may, in turn, serve as possible predictors of future cardiovascular events [4, 5]. Previous observational studies indicated that IMT in children and adolescents elevated with familial hypercholesterolemia [6], type 1 diabetes mellitus [7], and hypertension [8]. Measuring cIMT in children and adolescents with obesity has been recommended by the Association for European Paediatric Cardiology (AEPC), as it is capable of recognizing early vascular alterations and thus prevent myocardial infarction and stroke [9].

With regard to a possible relationship between IMT and insulin resistance/hyperinsulinemia in children and adolescents, the results remain inconsistent, with some studies reporting an adverse relation between insulin resistance and vascular measures $[10,11]$, while others observed no significant relations at all [12-14]. The studies in question did not look for differences based on gender or pubertal status.

By studying possible associations between insulin resistance and measures of vascular structure, while considering gender and pubertal maturation status, we may gain some insight on and advance our understanding of the complex gender-specific relations between obesity and vascular structure in response to insulin level. The present study has addressed this question by investigating possible associations between markers of insulin metabolism and cIMT among children and adolescents with either overweight or obesity.

\section{Methods}

\section{Study population}

This study was conducted during the baseline (cross-sectional) phase of a randomized, double-blind trial, aimed at investigating the effect of different doses of one-year vitamin D supplementation on CIMT and other bone and cardiovascular variables in children and adolescents with an age- and sex-specific body mass index (BMI) Z-score $\geq 1$ (according to the criteria established by the World Health Organization). Participants, aged 6 to 13 years, were recruited from primary schools located in three Tehran (Iran) districts. The baseline screening took place between June 2016 and March 2017. Individuals were eligible for inclusion if they had no known medical illnesses such as diabetes, liver, or kidney diseases, were not taking any dietary supplements, or using pharmaceutical agents that affect glucose and lipid metabolism. A total of 378 participants met these selection criteria and were enrolled at the baseline. The sample size for the trial was determined by a sample size calculation designed to achieve $80 \%$ power to detect a $0.02 \mathrm{~mm}$ difference in cIMT with a 0.05 standard deviation $(95 \%$ confidence interval) between different groups of the original trial.

\section{Measurements}

Fat and soft lean mass were assessed by portable bioelectrical impedance analyzer GAIA 359 PLUS 8-contact electrode bioelectrical impedance (BIA) system (Jawon Medical Co. Ltd., Shinsang, Korea). During the measurement, all participants removed their shoes and socks, wore light clothing, and stood with the soles of their feet making contact with the foot electrodes, while holding the hand electrodes in their bare hands. All necessary variables including sex, height, and age, were input into the instrument. The BIA system measured the impedance via a tetra-polar multi-frequency $(5,50,250 \mathrm{kHz})$ electrode method. Resistance to the $500 \mu \mathrm{A}$ current was measured while the participants stood motionless on the analyzer's platform, their arms raised about $30^{\circ}$, while lightly holding onto the hand electrodes. For the data interpretation, we used the manufacturer's software in its "standard" setting.

Body weight was measured using the scale function of the GAIA 359 PLUS (100-g accuracy) while the subjects were standing barefoot and wearing light clothing. Height was measured while participants were standing without shoes and shoulders in normal alignment, using a stadiometer with an accuracy of $0.5 \mathrm{~cm}$. Body mass index (BMI) was calculated as weight (in kilograms) divided by height (in meters) squared $\left(\mathrm{kg} / \mathrm{m}^{2}\right)$. Waist circumference (WC) was measured down to the nearest $0.5 \mathrm{~cm}$ at the level of the umbilicus, over light clothing, and without any pressure, using a tape meter.

Blood pressure was measured twice, at least $1 \mathrm{~min}$ apart, using a mercury sphygmomanometer and the Korotkoff sound technique, with an accuracy of $2 \mathrm{mmHg}$, after participants had been resting for $15 \mathrm{~min}$ on a chair; the average of both measurements served as the participant's final pressure; systolic/diastolic blood pressures (SBP/DBP) were determined from the first onset/disappearance of sound.

Blood samples were collected from all study participants between 8:00 and 10:00 AM, after 10-12 h of overnight fasting. All the blood analyses were carried out at the RIES research laboratory. Fasting blood glucose (FBG) was measured by enzymatic colorimetric method using glucose oxidase (Pars Azmoon, Tehran, Iran) and the Selectra 2 auto-analyzer (Vital Scientific, Spankeren, Netherlands) with intra- and inter-assay coefficients of variation $(\mathrm{CVs})$ of 1.1 and $1.4 \%$, respectively. Fasting serum insulin was determined by the electrochemiluminescence immunoassay (ECLIA) method, using Roche Diagnostics kits and the Roche/Hitachi Cobas e-411 analyzer (Roche Diagnostics, $\mathrm{GmbH}$, Mannheim, Germany). Intra- and inter-assay 
CVs were 1.3 and $2.5 \%$, respectively. To monitor the quality of measurements, assayed serum controls in different concentrations were used for the glucose (Pars Azmoon, Tehran, Iran) and insulin assays (Lyphochek Immunoassay Plus Control, Bio-Rad Laboratories).

Physical activity was assessed using the Modifiable Activity Questionnaire (MAQ) to calculate metabolic equivalent task minutes per week; the high reliability (97\%) and moderate validity (49\%) of the Persian translated MAQ in adolescents have been ascertained previously [15].

Pubertal status was classified according to the Tanner scale by a well-trained endocrinologist, dividing the participants into 2 groups based on breast and genital stages: pre-pubertal (boys at genital stage I, girls at breast stage I) and pubertal (boys at genital stage $\geq \mathrm{II}$, girls at breast stage $\geq \mathrm{II}$ ).

Carotid intima-media thickness was measured by a well-trained radiologist (P.D.). Participants were examined in the supine position with the head slightly extended and rotated away from the examiner. The carotid arteries were interrogated using a high-resolution Samsung ultrasound machine (model UGEO WS80A) with a linear-array transducer operating at a frequency of at least $7 \mathrm{MHz}$. Depth, gain, and focus was adjusted for each participant individually so that the arterial lumen was completely anechoic and in the center of the image. Common cIMT was measured from longitudinal B-mode images of the distal $1 \mathrm{~cm}$ of the far wall of each common carotid artery (CCA), between the intimal-luminal and the medial-adventitial interfaces of the carotid artery wall, represented as a double-line density on the ultrasound image. In rare cases where appropriate images of the distal CCA could not be obtained, proximal or mid-CCA images were used for IMT measurement. Measurements were performed using the automated edge-tracking software (automated IMT calculator) which obviated the need to perform manual measurements.

\section{Definitions}

Insulin resistance/sensitivity was calculated as follows:

Homeostatic model assessment of insulin resistance $($ HOMA-IR $)=[$ fasting insulin $(\mu \mathrm{U} / \mathrm{ml}) \times$ fasting glucose $(\mathrm{mmol} / \mathrm{l})] / 22.5$;

Quantitative insulin sensitivity check index (QUICKI) $=$ $1 /[\log$ fasting insulin $(\mu \mathrm{U} / \mathrm{ml})+\log$ fasting glucose $(\mathrm{mg} / \mathrm{dl})]$.

Abdominal obesity was defined as waist circumference (WC) $\geq 90$ th percentile for age and sex, according to national reference curves [16].

\section{Statistical analysis}

Quantitative variables were expressed as the mean \pm SD or median (interquartile range) while categorical variables were expressed as percentages. Continuous and categorical variables were compared according to sex using a t-test and a Chi square-test, respectively. Multiple linear regression analysis was used to evaluate the association of markers of insulin metabolism and cIMT. A proportional odds model (POM) was used to test for a possible association between an insulin metabolism marker increase of approximately $1 \mathrm{SD}$ and tertiles of cIMT using the ranges: cIMT $<0.38,0.38 \leq \mathrm{cIMT}<0.42$, and cIMT $\geq 0.42 \mathrm{~mm}$. The proportional odds assumption was assessed through a chi-squared score test for each covariate to examine whether the assumption was violated. Proportional odds assumptions were generally appropriate. To test whether pubertal stage or gender could affect the contribution of fasting insulin, the interaction terms for [female $\times$ fasting insulin] and [pre-pubertal $\times$ fasting insulin] were used as separate inputs in our multivariable linear regression models. The likelihood ratio test for interaction terms was not significant. Nevertheless, the interaction term for gender was of biological importance which is why each model was performed in the subset specified by gender. Waist circumference, body fat percentage, physical activity, and Tanner stages were considered as confounding variables. Collinearity was assessed using Pearson's rank correlation coefficient and variance inflation factor. No collinearity was found between body fat percentage and WC. All analyses were performed using IBM SPSS for Windows, version 20 (SPSS, Chicago, IL, USA) with the significance level set at $P<0.05$ (two-tailed).

\section{Results}

The study consisted of 378 participants (198 boys and 180 girls) with a mean $( \pm \mathrm{SD})$ age of $9.3 \pm 1.7$ years and $18.3 \%$ in the pre-pubertal stage. Compared to girls, boys had a higher physical activity, more hours of TV watching, a greater BMI, WC, soft lean mass, prevalence of obesity, a lower fat mass and HOMA-IR $(P<0.05$, Table 1$)$.

In boys, but not girls, both fasting insulin and HOMA-IR were positively, and QUICCKI negatively correlated with cIMT (Fig. 1). In boys, after controlling for body fat mass percentage, WC, physical activity, and Tanner stage, a one-SD increase in fasting insulin and HOMA-IR were associated with $0.351 \mathrm{~mm}(P<0.001)$ and $0.350 \mathrm{~mm}(P<0.001)$ increases in cIMT, respectively. However, a one-SD increase in QUICKI was associated with a $-0.305 \mathrm{~mm}(P=0.001)$ decrease in cIMT. When categorizing into tertiles, a one-SD increase in fasting insulin and HOMA-IR were associated with 87 and $81 \%$ increases in the odds of higher categories of cIMT (both $P<0.05$ ). However, a one-SD increase in QUICKI was associated with a 37\% lower odds of higher categories of cIMT $(P=0.022)$. No significant associations were found among girls (Table 2). 
Table 1 Characteristics of 378 overweight and obese children and adolescents

\begin{tabular}{|c|c|c|c|c|}
\hline & Total $(n=378)$ & Girls $(n=180)$ & Boys $(n=198)$ & $P$-value \\
\hline Age (years) & $9.3 \pm 1.7$ & $9.2 \pm 1.7$ & $9.4 \pm 1.8$ & 0.326 \\
\hline Pre-pubertal (\%) & 18.3 & 15.0 & 21.3 & 0.113 \\
\hline Physical activity (MET/hr./wk) & $13.3(5.1-31.7)$ & $8.9(3.3-17.9)$ & $20.1(7.9-41.2)$ & $<0.001$ \\
\hline \multicolumn{5}{|l|}{ Watching TV (hour/d) } \\
\hline$\leq 1$ & 14.1 & 14.2 & 13.9 & \multirow[t]{3}{*}{0.024} \\
\hline $2-3$ & 38.6 & 45.5 & 32.5 & \\
\hline$\geq 4$ & 47.3 & 40.3 & 53.6 & \\
\hline Height (cm) & $139.7 \pm 11.0$ & $138.9 \pm 10.9$ & $140.5 \pm 11.0$ & 0.142 \\
\hline Z-Score Height & $0.86 \pm 1.00$ & $0.80 \pm 1.07$ & $0.91 \pm 0.93$ & 0.291 \\
\hline BMI $\left(\mathrm{kg} / \mathrm{m}^{2}\right)$ & $23.3 \pm 3.4$ & $22.8 \pm 3.2$ & $23.8 \pm 3.4$ & 0.004 \\
\hline Z-Score BMI & $2.55 \pm 0.73$ & $2.34 \pm 0.83$ & $2.74 \pm 0.83$ & $<0.001$ \\
\hline Obese (\%) & 68.8 & 55.6 & 80.8 & $<0.001$ \\
\hline Waist circumference $(\mathrm{cm})$ & $80.8 \pm 9.5$ & $79.4 \pm 8.9$ & $82.0 \pm 10.0$ & 0.009 \\
\hline Abdominal obesity (\%) & 92.6 & 93.1 & 92.1 & 0.705 \\
\hline Fat mass (\%) & $26.3 \pm 6.9$ & $28.6 \pm 4.2$ & $24.2 \pm 8.1$ & $<0.001$ \\
\hline Soft-lean mass (kg) & $30.7 \pm 7.2$ & $29.1 \pm 6.6$ & $32.2 \pm 7.3$ & $<0.001$ \\
\hline Fasting glucose (mg/dl) & $90.7 \pm 9.3$ & $91.5 \pm 8.6$ & $89.9 \pm 9.9$ & 0.103 \\
\hline Fasting insulin $(\mu \mathrm{U} / \mathrm{ml})$ & $12.4(8.5-19.0)$ & $12.9(9.3-20.6)$ & $11.9(7.9-18.2)$ & 0.085 \\
\hline HOMA-IR & $2.80(1.92-4.34)$ & $3.00(2.02-4.56)$ & $2.66(1.67-4.13)$ & 0.048 \\
\hline QUICKI & $0.333 \pm 0.040$ & $0.328 \pm 0.031$ & $0.338 \pm 0.046$ & 0.023 \\
\hline Carotid intima media thickness (mm) & $0.40 \pm 0.057$ & $0.40 \pm 0.004$ & $0.41 \pm 0.060$ & 0.100 \\
\hline
\end{tabular}

BMI body mass index, HOMA-IR homeostatic model assessment of insulin resistance, QUICKI quantitative insulin sensitivity check index Quantitative variables were expressed as the mean \pm SD or median (interquartile range) and categorical ones as percentages Abdominal obesity was defined based on national age- and sex-specific 90th percentile (1)

\section{Discussion}

This study demonstrated that markers of insulin metabolism were independent predictors of cIMT in overweight and obese boys, but not in girls. Both fasting insulin and HOMA-IR were positively, and QUICKI negatively associated with cIMT. This relationship remained significant even after adjusting for obesity features such as body fat percentage and WC, or pubertal status (Tanner stages). This result not only highlights the robustness of the link between insulin resistance and cIMT as an independent risk factor for the development of atherosclerosis, but it clearly underscores how arterial abnormalities are demonstrable already at a very early age in overweight and obese boys.

A number of observational studies among adults have shown adverse structural changes in cIMT in the presence of cardiovascular (CVD) risk factors [17, 18]. However, data regarding subclinical CVD using imaging such as cIMT in pediatrics with overweight and obesity is limited and inconsistent. While some studies reported that increased cIMT was mediated in part by obesity [19], insulin sensitivity [20], type 2 diabetes [21], DBP [22], SBP, inflammatory markers [23], epicardial adipose tissue thickness [24], WC [22, 25], others found no associations between cIMT and insulin resistance [14, 26], prediabetes state [27], polycystic ovary syndrome [28], and inflammatory chemokines [29].

Our findings are in agreement with results of recent investigations where higher insulin levels and the presence of insulin resistance were adversely associated with carotid wall thickness among children and adolescents. Ryder et al. (2016) conducted a cross-sectional study on 252 children, aged between 8 and 20 years and in Tanner stages ranging from 2 to 5 , and observed that cIMT was positively related to insulin resistance (measured by hyperinsulinemic euglycemic clamp) [10]. In addition, type 2 diabetes, a state of insulin resistance, was associated with higher cIMT in youth aged 10-24 years [21]. Furthermore, Atabek et al. observed in obese children aged between 8 and 18 years that QUICKI was independently associated with cIMT, but not HOMA-IR [30]. However, Reinehr et al. did not find any significant correlation between insulin level and cIMT among 96 obese children with a mean age of 11 years, after controlling for pubertal status and body fat mass [23]. Besides, there was no significant difference with regard to IMT between obese insulin resistant and obese not insulin resistant children aged 12 to 18 years, a fact that 

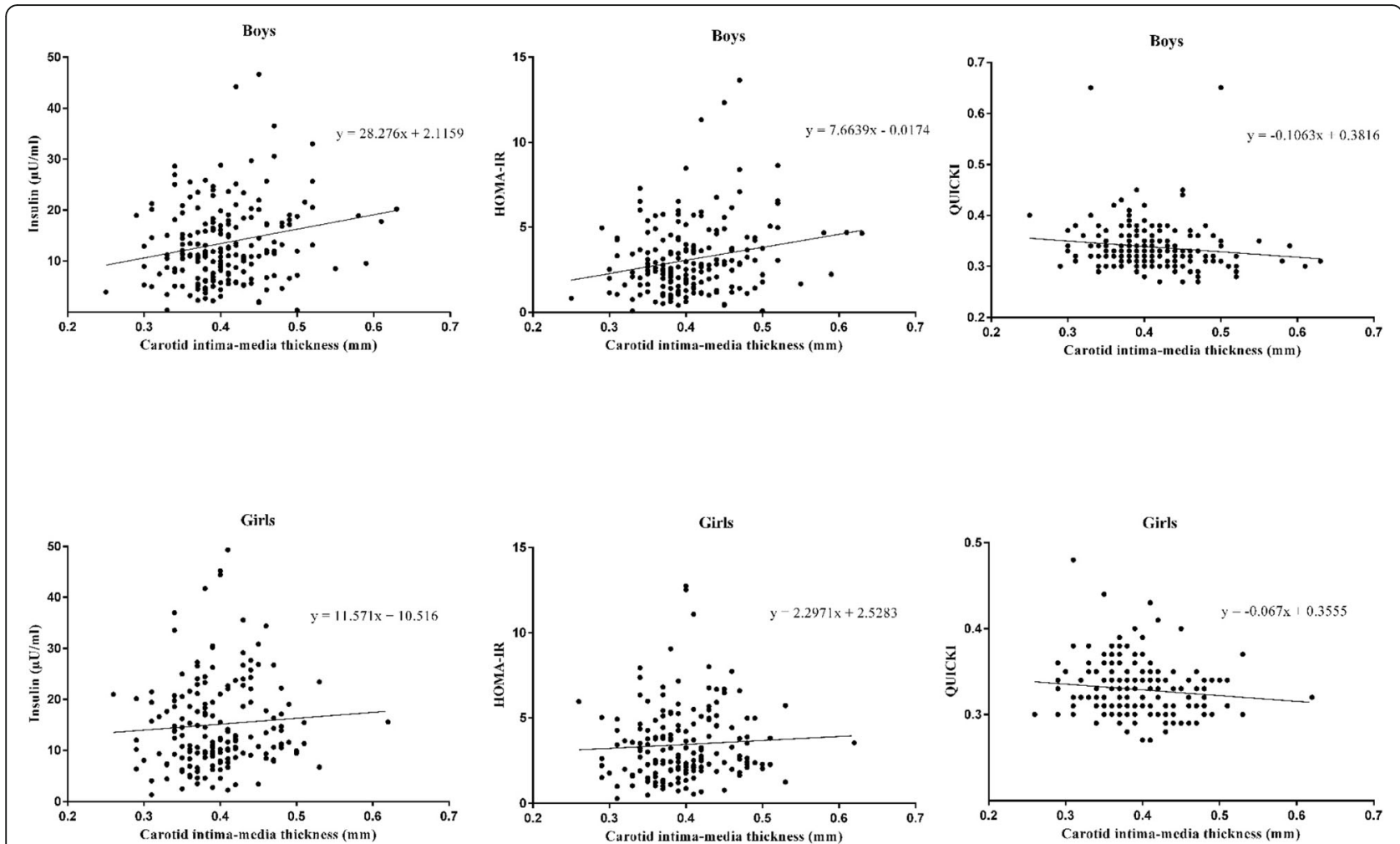

Fig. 1 Gender-specific correlations of fasting insulin, homeostatic model assessment of insulin resistance (HOMA-IR), and quantitative insulin sensitivity check index (QUICKI) with carotid intima-media thickness among 378 overweight and obese children and adolescents

could be due to the $\beta$-error associated with the sample size [14]. Compared to most previous studies, our study had a larger sample size, narrower age range, and accounted for potential confounding variables (physical activity, body fat percentage, WC, and pubertal status). In contrast to these studies, our findings clearly indicate how markers of insulin metabolism are associated with carotid atherosclerosis, and we found this association to be independent of adiposity. These differences may be due to the fact that we considered an overweight to an obese pediatric population with a narrower BMI than most other studies. In addition, these differences may also be partly due to differences in the protocol used for measuring cIMT.

Another difference compared to previous studies is that we separated our participants by gender. This yielded the somewhat surprising result that the association between insulin-related variables and cIMT exists only among boys but not among girls. The reason for this finding is not clear; there is a difference between girls and boys regarding weight status and WC values. Lower obesity prevalence and WC among girls compared to these among boys may be an explanation to justify lack of association between insulin resistance and IMT in girls. It might also explain the higher susceptibility of boys to developing CVD and the fact that CVD events occur approximately 10 years earlier in life for men that for women [31]. Increased adiposity, in combination with higher glucose and insulin levels during childhood, has been linked to a higher carotid thickness in later life [12, 30]. The Bogalusa Heart Study found that the cumulative levels of high BMI in childhood was associated with adult cIMT in both sexes, even after controlling for adult BMI [32]. Increased body weight may interact with insulin level and sensitivity by affecting atherosclerosis.

Several studies illustrated an association between obesity and thicker cIMT among children and adolescents. The significance of this relation became marginal once the model applied to 100 children with BMI $>95$ th percentile was adjusted for glucose [19]. Besides, Reinehr et al. revealed that impaired fasting glucose, along with BMI, was an independent determinant of cIMT in obese children. Their results indicate the importance of impaired glucose hemostasis for the arterial structure during childhood and even adulthood [12]. Furthermore, the linkage of insulin resistance and increased cIMT may be explained by the atherogenic pattern of lipoprotein particles and higher endothelial-derived ICAM, which are both present among obese insulin-resistant adolescents [14].

By surveying a large number of children and adolescents, we could observe associations with regard to 
Table 2 Associations of insulin metabolism markers with carotid intima media thickness according to sex

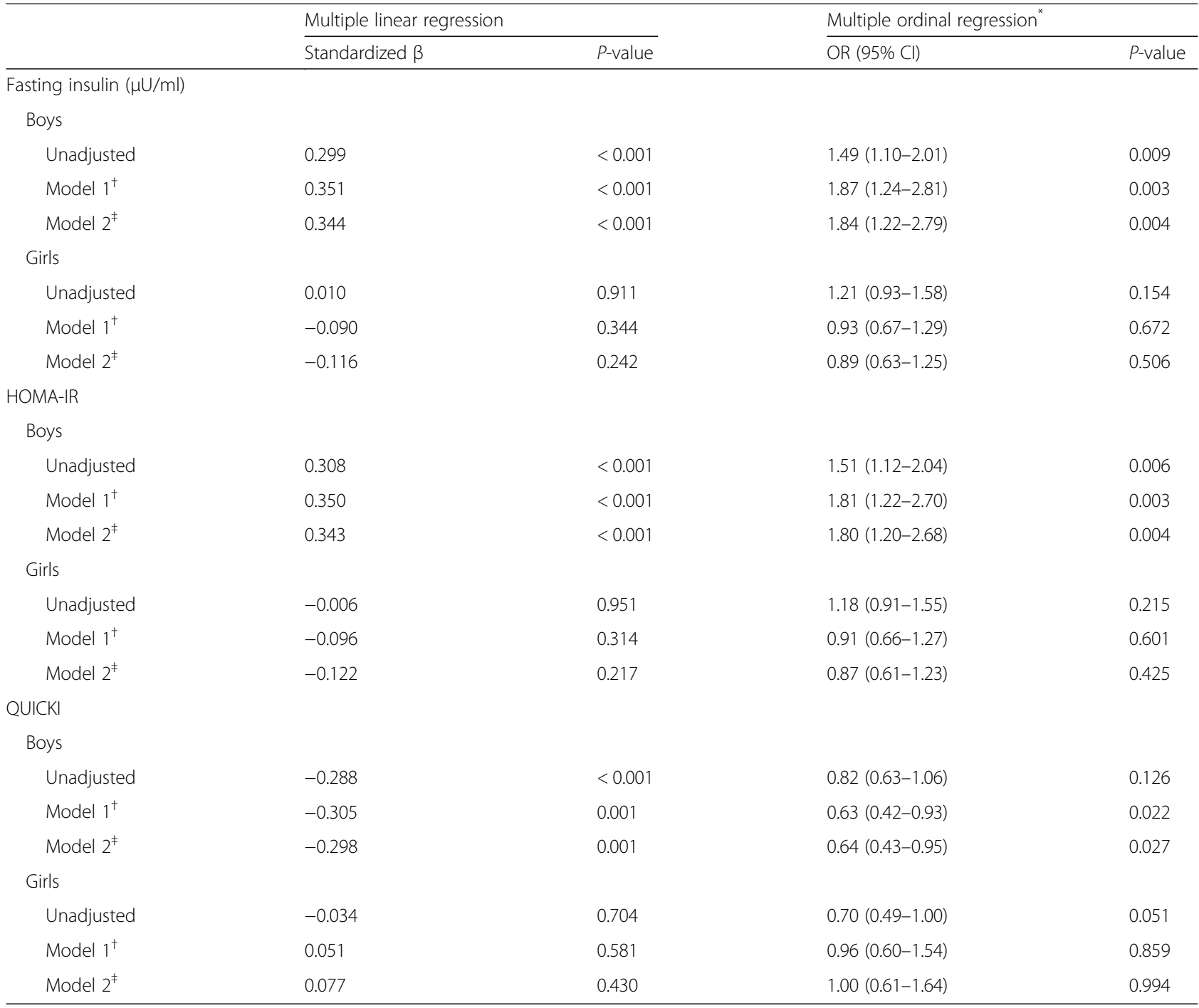

HOMA-IR homeostatic model assessment of insulin resistance, QUICKI quantitative insulin sensitivity check index

"Multiple ordinal regression was used with carotid intima media thickness as response with three ordered categories

${ }^{+}$Adjusted for body fat mass percent, waist circumference, and physical activity

${ }^{\ddagger}$ Additionally adjusted for Tanner stages

gender. BIA measurements allowed us to adjust for adiposity (body fat percentage) instead of only BMI. Despite these strengths, our study also has some inherent limitations: the participants came from a narrowly localized geographic region (the southern districts of Tehran), which may limit generalizability; due to this study having been strictly observational, causality could be established; despite measurement of a number of confounders, residual confounding could not be ruled out. Assessing breast development among overweight and obese girls may be overestimated because of difficulties in distinguishing between glandular breast tissue and subcutaneous fat; however, in the current study, a welltrained woman endocrinologist was used to visual assessment of breast budding (Tanner stage 2 development) which minimized measurement error. And lastly, our interpretation of insulin resistance is predominantly based on the HOMA-IR and not on euglycaemic clamp studies, which was simply not possible given our study parameters.

\section{Conclusion}

In conclusion, the current study showed that HOMA-IR and QUICKI, as a markers of insulin resistance and sensitivity, may serve as independent predictors of cIMT in overweight and obese boys, but not in girls. Our study provides biologically important information and further work is recommended to translate these findings into clinical practice for identifying high-risk children. 


\section{Abbreviations}

BIA: bioelectrical impedance; BMI: body mass index; CCA: common carotid artery; CIMT: Carotid intima media thickness; DBP: diastolic blood pressure; FBG: fasting blood glucose; HOMA-IR: homeostatic model assessment of insulin resistance; POM: proportional odds model; QUICKI: quantitative insulin sensitivity check index; SBP: systolic blood pressure; SD: standard deviation; WC: waist circumference

\section{Acknowledgments}

We would like to acknowledge Ms. Niloofar Shiva for a critical edition of English grammar and syntax of the manuscript.

\section{Funding}

This work was funded by a grant from the Faculty of Nutrition Sciences and Food Technology, National Nutrition and Food Technology Research Institute, in addition to the Research Institute for Endocrine Sciences, Shahid Beheshti University of Medical Sciences, Tehran, Iran. The funder were not involved in the design of the study and collection, analysis, and interpretation of data and in writing the manuscript.

\section{Availability of data and materials}

The datasets analyzed during the current study are available from the corresponding author on reasonable request.

\section{Authors' contributions}

All authors have read and approved the final manuscript. Overall P. M., F. H. and F. A. supervised the project and approved the final version of the manuscript to be submitted. F. H., G. A. and E. Y. designed the research; M. T. and T. N. assessed the laboratory and biochemical parameters; P. D. measured carotid intima-media thickness; P. M., M. M. and G. A., analyzed and interpreted the data, and G. A., E.Y., and F. H. drafted the initial manuscript; F. A. critically revised the manuscript.

\section{Ethics approval and consent to participate}

Parents gave written informed consent and all children provided assent to participate. Ethics approval was obtained from the ethics committee of the Research Institute for Endocrine Sciences, Shahid Beheshti University of Medical Sciences (NO: IR.SBMU.ENDOCRINE.REC.1395.373).

\section{Consent for publication}

Not applicable.

\section{Competing interests}

The authors declare that they have no competing interests.

\section{Publisher's Note}

Springer Nature remains neutral with regard to jurisdictional claims in published maps and institutional affiliations.

\footnotetext{
Author details

${ }^{1}$ Department of Clinical Nutrition and Dietetics, Faculty of Nutrition Sciences and Food Technology, National Nutrition and Food Technology Research Institute, Shahid Beheshti University of Medical Sciences, Tehran, Iran. ${ }^{2}$ Department of Imaging, Research Development Center, Taleghani Hospital, Shahid Beheshti University of Medical Sciences, Tehran, Iran. ${ }^{3}$ Nutrition and Endocrine Research Center, Research Institute for Endocrine Sciences, Shahid Beheshti University of Medical Sciences, P.O. Box: 19395-4763, Tehran, Iran. ${ }^{4}$ Nutrition and Endocrine Research Center, Research Institute for Endocrine Sciences, Shahid Beheshti University of Medical Sciences, Tehran, Iran. ${ }^{5}$ Obesity Research Center, Research Institute for Endocrine Sciences, Shahid Beheshti University of Medical Sciences, Tehran, Iran. ${ }^{6}$ Prevention of Metabolic Disorders Research Center, Research Institute for Endocrine Sciences, Shahid Beheshti University of Medical Sciences, Tehran, Iran. ${ }^{7}$ Laboratory of Nutrition Research, National Nutrition and Food Technology Research Institute, Faculty of Nutrition Sciences and Food Technology, Shahid Beheshti University of Medical Sciences, Tehran, Iran. ${ }^{8}$ Obesity Research Center, Research Institute for Endocrine Sciences, Shahid Beheshti University of Medical Sciences, P.O. Box: 19395-4763, Tehran, Iran. ${ }^{9}$ Endocrine Research Center, Research Institute for Endocrine Sciences, Shahid Beheshti University of Medical Sciences, Tehran, Iran.
}

Received: 7 March 2018 Accepted: 16 November 2018

Published online: 23 November 2018

\section{References}

1. Sinaiko AR, Caprio S. Insulin resistance. J Pediatr. 2012;161(1):11-5.

2. Perkins JM, Joy NG, Tate DB, Davis SN. Acute effects of hyperinsulinemia and hyperglycemia on vascular inflammatory biomarkers and endothelial function in overweight and obese humans. American Journal of PhysiologyEndocrinology and Metabolism. 2015;309(2):E168-76.

3. Rosvall M, Persson M, Östling G, Nilsson P, Melander O, Hedblad B, Engström $\mathrm{G}$. Risk factors for the progression of carotid intima-media thickness over a 16-year follow-up period: the Malmö diet and Cancer study. Atherosclerosis. 2015;239(2):615-21.

4. Kuller L, Borthani N, Furberg C, Gardin J, Manolio T, O'leary D, Psaty B, Robbins J. Prevalence of subclinical atherosclerosis and cardiovascular disease and association with risk factors in the cardiovascular health study. Am J Epidemiol. 1994;139(12):1164-79.

5. Rahman TJ, Walker EA, Mayosi BM, Hall DH, Avery PJ, Connell JM, Watkins H, Stewart PM, Keavney B. Genotype at the P554L variant of the hexose-6 phosphate dehydrogenase gene is associated with carotid intima-medial thickness. PLoS One. 2011;6(8):e23248.

6. Tonstad S, Joakimsen O, Stensland-Bugge E, Leren TP, Ose L, Russell D, Bønaa $\mathrm{KH}$. Risk factors related to carotid intima-media thickness and plaque in children with familial hypercholesterolemia and control subjects. Arterioscler Thromb Vasc Biol. 1996;16(8):984-91.

7. Järvisalo MJ, Putto-Laurila A, Jartti L, Lehtimäki T, Solakivi T, Rönnemaa T, Raitakari OT. Carotid artery intima-media thickness in children with type 1 diabetes. Diabetes. 2002;51(2):493-8.

8. Lande MB, Carson NL, Roy J, Meagher CC. Effects of childhood primary hypertension on carotid intima media thickness. Hypertension. 2006; 48(1):40-4.

9. Dalla Pozza R, Ehringer-Schetitska D, Fritsch P, Jokinen E, Petropoulos A, Oberhoffer R. Intima media thickness measurement in children: a statement from the Association for European Paediatric Cardiology (AEPC) working group on cardiovascular prevention endorsed by the Association for European Paediatric Cardiology. Atherosclerosis. 2015;238(2):380-7.

10. Ryder JR, Dengel DR, Jacobs DR Jr, Sinaiko AR, Kelly AS, Steinberger J. Relations among adiposity and insulin resistance with flow-mediated dilation, carotid intima-media thickness, and arterial stiffness in children. J Pediatr. 2016;168:205-11.

11. Woo KS, Chook P, Yu CW, Sung RY, Qiao M, Leung SS, Lam CW, Metreweli C, Celermajer DS. Overweight in children is associated with arterial endothelial dysfunction and intima-media thickening. Int J Obes Relat Metab Disord. 2004;28(7):852-7.

12. Yajnik CS, Katre PA, Joshi SM, Kumaran K, Bhat DS, Lubree HG, Memane N, Kinare AS, Pandit AN, Bhave SA, et al. Higher glucose, insulin and insulin resistance (HOMA-IR) in childhood predict adverse cardiovascular risk in early adulthood: the Pune Children's study. Diabetologia. 2015; 58(7):1626-36

13. Gazolla FM, Neves Bordallo MA, Madeira IR, de Miranda Carvalho CN, Vieira Monteiro AM, Pinheiro Rodrigues NC, Borges MA, Collett-Solberg PF, Muniz $\mathrm{BM}$, de Oliveira CL. Association between cardiovascular risk factors and carotid intima-media thickness in prepubertal Brazilian children. J Pediatr Endocrinol Metab. 2015:28(5-6):579-87.

14. Brar PC, Patel P, Katz S. The relationship between insulin resistance and endothelial dysfunction in obese adolescents. J Pediatr Endocrinol Metab. 2017;30(6):635-42

15. Delshad M, Ghanbarian A, Ghaleh NR, Amirshekari G, Askari S, Azizi F. Reliability and validity of the modifiable activity questionnaire for an Iranian urban adolescent population. Int J Prev Med. 2015;6:3.

16. Kelishadi R, Gouya MM, Ardalan G, Hosseini M, Motaghian M, Delavari A Majdzadeh R, Heidarzadeh A, Mahmoud-Arabi MS, Riazi MM. First reference curves of waist and hip circumferences in an Asian population of youths: CASPIAN study. J Trop Pediatr. 2007:53(3):158-64.

17. O'Leary DH, Polak JF, Kronmal RA, Manolio TA, Burke GL, Wolfson SK Jr. Carotid-artery intima and media thickness as a risk factor for myocardial infarction and stroke in older adults. N Engl J Med. 1999:340(1):14-22.

18. Simon A, Megnien J-L, Chironi G. The value of carotid intima-media thickness for predicting cardiovascular risk. Arterioscler Thromb Vasc Biol. 2010;30(2):182-5. 
19. Iannuzzi A, Licenziati MR, Acampora C, Salvatore V, Auriemma L, Romano ML, Panico S, Rubba P, Trevisan M. Increased carotid intima-media thickness and stiffness in obese children. Diabetes Care. 2004;27(10):2506-8.

20. Rathsman B, Rosfors S, Sjoholm A, Nystrom T. Early signs of atherosclerosis are associated with insulin resistance in non-obese adolescent and young adults with type 1 diabetes. Cardiovasc Diabetol. 2012;11:145.

21. Urbina EM, Kimball TR, McCoy CE, Khoury PR, Daniels SR, Dolan LM. Youth with obesity and obesity-related type 2 diabetes mellitus demonstrate abnormalities in carotid structure and function. Circulation. 2009;119(22):2913-9.

22. Elkiran O, Yilmaz E, Koc M, Kamanli A, Ustundag B, Ilhan N. The association between intima media thickness, central obesity and diastolic blood pressure in obese and overweight children: a cross-sectional school-based study. Int J Cardiol. 2013;165(3):528-32.

23. Reinehr T, Kiess W, de Sousa G, Stoffel-Wagner B, Wunsch R. Intima media thickness in childhood obesity: relations to inflammatory marker, glucose metabolism, and blood pressure. Metabolism. 2006;55(1):113-8.

24. Elshorbagy HH, Fouda ER, Kamal NM, Bassiouny MM, Fathi WM. Evaluation oF Epicardial fat and carotid intima-media thickness in obese children. Iran J Pediatr. 2016;26(1):e2968.

25. Melo X, Santa-Clara H, Pimenta NM, Carrolo M, Martins SS, Minderico CS, Fernhall B, Sardinha LB. Body composition phenotypes and carotid intimamedia thickness in 11-13-year-old children. Eur J Pediatr. 2014;173(3):345-52.

26. Miniello VL, Faienza MF, Scicchitano P, Cortese F, Gesualdo M, Zito A, Basile $M$, Recchia $P$, Leogrande $D$, Viola $D$, et al. Insulin resistance and endothelial function in children and adolescents. Int J Cardiol. 2014;174(2):343-7.

27. Shah AS, Gao Z, Urbina EM, Kimball TR, Dolan LM. Prediabetes: the effects on arterial thickness and stiffness in obese youth. J Clin Endocrinol Metab. 2014;99(3):1037-43.

28. Hughan KS, Tfayli H, Warren-Ulanch JG, Barinas-Mitchell E, Arslanian SA. Early biomarkers of subclinical atherosclerosis in obese adolescent girls with polycystic ovary syndrome. J Pediatr. 2016;168:104-111.e101.

29. Eikendal AL, Evelein AM, Uiterwaal CS, van der Ent CK, Visseren FL, Bots ML, Hoefer IE, den Ruijter HM, Dalmeijer GW. Relation between circulating inflammatory chemokines and vascular characteristics in healthy, young children. J Am Heart Assoc. 2015;4(12).

30. Atabek ME, Pirgon O, Kivrak AS. Evidence for association between insulin resistance and premature carotid atherosclerosis in childhood obesity. Pediatr Res. 2007;61(3):345-9.

31. Marmot MG, Elliott P: Coronary heart disease epidemiology: from aetiology to public health: Oxford Medical Publications; 2005.

32. Freedman DS, Patel DA, Srinivasan SR, Chen W, Tang R, Bond MG, Berenson GS. The contribution of childhood obesity to adult carotid intima-media thickness: the Bogalusa heart study. Int J Obes. 2008;32(5):749-56.

Ready to submit your research? Choose BMC and benefit from:

- fast, convenient online submission

- thorough peer review by experienced researchers in your field

- rapid publication on acceptance

- support for research data, including large and complex data types

- gold Open Access which fosters wider collaboration and increased citations

- maximum visibility for your research: over $100 \mathrm{M}$ website views per year

At $\mathrm{BMC}$, research is always in progress.

Learn more biomedcentral.com/submissions 